DOI: $10.26565 / 2220-7929-2019-55-03$

УДК 930.1:378.4-054.62(430)

\title{
Quellen und Forschungen zur Geschichte des Ausländerstudiums in Deutschland. Kritische Bemerkungen zu zentralen Problemen
}

\author{
Hartmut Rüdiger Peter
}

\author{
https://orcid.org/0000-0001-9331-7290 \\ Doctor of Sciences (History), Professor \\ Martin Luther University Halle-Wittenberg \\ 6 Emil-Abderhalden-Str., 06108, Halle (Saale), Germany \\ hartmut.peter@geschichte.uni-halle.de
}

The article is devoted to the analysis of sources and literature on the history of the education of foreign students in German higher education institutions. The main goal of the article is to detect the most significant aspects of the research subject area and its impact on further investigations on history of universities. We note that the education of foreign students in Germany has provided to significant influence in the political discourse of this country. At the same time, various investigations practically ignore the issues related to our research subject area. The discussion of the last third of the XIX century in Germany about the excessive number of foreign students in German higher education institutions is considered. The discussion was called «akademische Ausländerfrage» and appeared together with the «overcrowding crisis» by foreign students from technical higher education institutions and medical faculties of German universities. Two issues were central in the discussion. On the one hand, nationalist and anti-Semitic groups strongly opposed a large number of Jews from Eastern Europe among students of German universities. Moreover, Polish and Russian students were also considered as «undesirable foreigners». The principle of internationality of universities was subjected to attacks and discredit as fundamental for the academic community of Europe. On the other hand, the universities strongly protested against interference in their internal affairs and their autonomy in general. In particular German universities strongly opposed any restrictions on foreign students obtaining university education. However universities were interested party in limiting the inflow of students with insufficient education and motivation. We conclude that the state policy of foreign students was equally opposed and harmonized the position of German universities.

Keywords: Germany, German universities, foreign students, academic community.

A. Quellen 1. Besonderheiten der Quellenlage in Deutschland. 1.1. Die Hochschulpolitik gehörte in Deutschland auch nach der Reichsgründung 1871 in die Kompetenz der Bundesstaaten. Daher gab es weder im Kaiserreich noch in der Weimarer Republik für den Gesamtbereich zuständige Reichsinstanzen. Kleinere deutsche 
Staaten orientierten sich zwar häufig am Beispiel Preußens, das über die größte Zahl höherer Lehranstalten verfügte, ohne dabei aber das ,preußische Modell“ einfach zu übernehmen. Für die reichsweite Harmonisierung sorgten Institutionen wie die „Konferenz von Vertretern deutscher Regierungen in Hochschulangelegenheiten" (1898-1933). Die Hochschulen und die Hochschullehrer setzten den staatlichen Regulierungsinstanzen mit der „Außeramtlichen Deutschen Rektorenkonferenz“ (seit 1903), dem „Deutschen Hochschullehrertag“ und dem „Verband deutscher Hochschulen“ eigene Beratungsgremien und Standesorganisationen entgegen. Nach dem Ende des Ersten Weltkrieges übernahm die Kulturabteilung des Auswärtigen Amtes (1920 eingerichtet) koordinierende Aufgaben bei der internationalen Hochschulzusammenarbeit und der Regulierung des Ausländerund Auslandsstudiums. Erst nach der nationalsozialistischen Machtergreifung wurde 1934 die Kulturhoheit der Länder mit der Bildung eines Reichsministeriums aufgehoben, das mit dem preußischen Kultusministerium zum „Reichs- und Preußischen Ministerium für Wissenschaft, Erziehung und Volksbildung“ fusionierte.

1.2. Die deutschen Universitäten waren zwar „Staatsanstalten“ in dem Sinne, dass sie vom Staat unterhalten wurden und sich einem immer dichter werdenden staatlichen Regelwerk unterworfen sahen. Gleichzeitig gestalteten sie ihre inneren akademischen Angelegenheiten weitgehend in Selbstverwaltung. Die Entscheidung über die Immatrikulation von Studierenden lag im Regelfall in den Händen der Hochschulen. Dies betraf auch die ausländischen Studienbewerber. Der Ermessensspielraum war dabei in den von den Kultusministerien genehmigten Hochschulstatuten und ggf. wie in Preußen musterhaft in allgemeinen „Vorschriften für die Studierenden der Landesuniversitäten“ festgeschrieben. Die überwiegend in der 2. Hälfte des 19. Jahrhunderts geschaffenen Technischen Hochschulen (TH) konnten in ihrer kurzen Geschichte keine ähnlich starken Traditionen der Selbstverwaltung ausprägen und waren direkter der Regulierungsgewalt des Staates unterworfen. In Preußen wurde so um 1900 die Zulassung ausländischer Studierender an den TH grundsätzlich geregelt. Andere Hochschulen - etwa die Landwirtschafts- und Forstakademien - unterstanden nicht den Kultusministerien, sondern den entsprechenden Fachministern, die ihre Immatrikulationspraxis an die Regelungen der Kultusministerien anlehnten.

Somit wurde die Politik gegenüber ausländischen Studierenden an den deutschen Hochschulen in einem Mit- und auch Gegeneinander verschiedener Institutionen mit jeweils eigenen Interessen ausgehandelt. Das relevante amtliche Schriftgut ist nicht zentralisiert, sondern überwiegend in drei Gruppen von Archiven - den Hochschularchiven, den Staatsbzw. Landesarchiven und den Archiven von Reichsinstitutionen - zu finden. Worauf der Schwerpunkt der Recherche zu legen ist, hängt von der jeweiligen Fragestellung ab.

2. Universitäts- und Hochschularchive. 2.1. Für personenbezogene Forschungen, in denen die nationale, soziale und konfessionelle Herkunft, die Studieninteressen, das soziale und gesellschaftliches Leben der Bildungsmigranten im Deutschen Reich im Mittelpunkt stehen, sind die Akten der Universitäts- und Hochschularchive von zentraler Bedeutung. Den Matrikelbücher oder -listen, Alben, Anmelde- und Adressbüchern oder Gasthörerverzeichnissen können wesentliche Daten zur Person und zum Studienverlauf entnommen werden, auf deren Grundlage es möglich ist, ausgewählte Gruppen von Studierenden in ihrer Zusammensetzung und ihren akademischen Interessen mit sozialstatistischen Methoden zu beschreiben. Die Führung solcher Verzeichnisse war allerdings in den deutschen Hochschulstaaten nicht einheitlich, so dass ihr Quellenwert unterschiedlich zu bewerten ist. Umfangreichen Angaben 
in Preußen, aber auch in Sachsen und Thüringen stehen eher spärliche Daten in Baden oder Bayern gegenüber. An der Universität Freiburg in Baden war zum Beispiel ganz in der Tradition selbstverständlicher Internationalität der Wissenschaft lediglich der Geburtsort des Studierenden anzugeben. Da zudem nur die zuletzt besuchte Lehranstalt genannt werden musste - häufig bereits eine Hochschule im Ausland - lassen sich Herkunft und wissenschaftliche Vorbildung nur schwer bewerten. Wenn Alben oder Anmeldebücher nicht überliefert sind, muss auf die gedruckten Verzeichnisse der Studierenden zurückgegriffen werden. Ergänzende Angaben über die Studienverläufe können je nach Überlieferung aus Studentenakten, Exmatrikeln oder Studienund Sittenzeugnisse gewonnen werden. An Technischen Hochschulen erfüllen die Diplomakten eine ähnliche Funktion. In den Fakultätsakten der Universitäten findet sich ebenfalls Schriftgut über Abschlussprüfungen und Promotionsverfahren mit personenbezogenen Angaben.

2.2. Allgemeine Fragen der Politik der jeweiligen Hochschulen gegenüber dem Studium von Ausländern sind in den Akten von Rektorat und Senat, in Preußen auch der Kuratoren als staatliche Aufsichtsbeamte bei den Universitäten, sowie in den Fakultätsakten zu recherchieren. Hier finden sich u.a. auch der Schriftwechsel mit den Kultusministerien, Petitionen deutscher Studenten, Stellungnahmen der Institute und einzelner Professoren. Allein schon der Kontext und der Zeitpunkt der Anlage spezieller Akten zum Ausländerstudium lassen Rückschlüsse auf die jeweilige Wahrnehmung der akademischen Ausländerfrage zu. Darüber hinaus spiegeln sie das inneruniversitäre Meinungsbild sowie differierende Interessen und Auffassungen wieder. An der Universität Heidelberg z.B. wurde Schriftgut über die ,Zulassung von Ausländern zur Immatrikulation“ bereits seit 1838 geführt, im benachbarten Freiburg erst seit 1907. Die meisten Bestände entstanden mit der Zuspitzung der Diskussionen um das Ausländerstudium im Reich um 1900. Die Fakultätsakten enthalten, je nach den örtlichen Gegebenheiten, oft auch Anfragen von Ausländern über Zulassungsvoraussetzungen, aus denen auch Auskünfte über ihre Vorbildung und die Motive für ein Studium in Deutschland erschlossen werden können. Interessant sind besonders stark nachgefragte Institute, an denen der überproportionalen Präsenz von Ausländern ein intensiverer Meinungsbildungsprozess stattfand.

2.3. Eine weitere Quelle sind die Akten zu den akademischen Vereinen ausländischer Studierender. Soweit diese nur Studierende der jeweiligen Hochschule als Mitglieder aufnahmen, fielen sie unter die akademische Gerichtsbarkeit, und es existiert Schriftgut in den Beständen des Rektors und Senats oder des Universitätsrichters. Wenn Vereine hochschulübergreifend waren oder der nichtakademischen Gesellschaft offen standen, unterlagen sie der Zuständigkeit der Polizeibehörden. In einigen deutschen Staaten, etwa in Bayer, werden Akten zu studentischen Vereinen auch in den Beständen der Polizeipräsidiums geführt. Die Dokumente in den Universitätsarchiven enthalten meist neben dem Ersuchen um Zulassung lediglich Statuten, Listen der Gründungsmitglieder und für die folgenden Semester Mitglieder- und Vorstandsverzeichnisse. SeltenersindProtokollederUniversitätsbehördenzu VernehmungenoderSpitzelberichtezufinden. $\mathrm{Da}$ ausländische Studenten in deutschen wissenschaftlichen Vereinen und - z.B. bei deutscher Abstammung - mitunter auch in einheimischen Studentenverbindungen mitwirkten, empfiehlt es sich, deren Akten gleichfalls durchzusehen.

3. Archive der deutschen Bundesstaaten. Schriftgut zum Ausländerstudium ist in den Beständen aller Ressorts zu finden, die sich mit seiner Organisation, politischen Regulierung, Überwachung und Finanzierung zu befassen hatten. Die Akten enthalten Berichte der Hochschulen, Antworten auf Umfragen zum Studium von Ausländern, statistische Zusammenstellungen, Namenslisten von Studenten, Sammlungen von Zeitungsausschnitten usw. Sie reflektieren politische Grundsatzdebatten über das Ausländerstudium, die Vorbereitung von Berichten in den Landtagen, den innerdeutschen Informations- und Meinungsaustausch, 
Verhandlungen zwischen den Ministerien. Struktur und Umfang der Bestände unterscheiden sich je nach der Organisation der Landeskabinette und der Relevanz der Hochschulpolitik.

3.1. In den Akten der Kultusministerien sind in der Regel eigenständige Bestände zum Studium von Ausländern zu finden, später ergänzt um das Auslandsstudium. Im Preußischen Geheimen Staatsarchiv in Berlin-Dahlem ist die Aktenführung aus historischen Gründen und entsprechend der internen Kompetenzverteilung zweigeteilt: Seit 1889 gibt für die Technischen Hochschulen separates Schriftgut über das Studium von Ausländern; der Bestand wurde bis 1925 geführt. Parallel dazu existieren seit 1897 „Acta betreffend die Zulassung von Ausländern zum Studium an den Universitäten“, die für die Zeit nach 1900 von maßgeblicher Bedeutung sind. Im Generallandesarchiv Karlsruhe gibt es 1902 beginnende Akten über die „Zulassung von Ausländern zum Studium an den badischen Hochschulen“ und über die „Aufnahme russischer Studierender an der Technischen Hochschule“. Das Sächsische Hauptstaatsarchiv Dresden führte gemeinsame Bestände für die Universität Leipzig und die TH Dresden. Im Bayerischen Hauptstaatsarchiv München sind die Quellen zum Ausländerstudium an den Universitäten eher dürftig. Ausführlicher ist die Dokumentation über die „Aufnahme der Studierenden, Zuhörer, Hospitanten und Ausländer“ der TH München, der Bestand wurde seit 1899 geführt. In den Thüringischen Landesarchiven ging ein Großteil der Akten des Kultusministeriums im Krieg verloren. Neben solchen Generalakten können auch andere Bestände von Interesse sein, z.B. für Preußen, wo das Ausländerstudium seit den 1890er Jahren auch im Zusammenhang mit der Reform des Medizinalwesens und dem Frauenstudium diskutiert wurde.

3.2. Soweit die ausländischen Studierenden politisch missliebiger Stimmungen und Aktivitäten oder der Verbindung zu politischen Exilorganisationen verdächtigt wurden, waren sie Gegenstand der Beobachtung durch die Innen- und Polizeibehörden. Dies traf in besonderem Maße auf Studierende aus Osteuropa und hier wiederum aus dem Russischen Reich zu. Aus diesem Grunde befinden sich auch in den Akten der Innenministerien und Polizeipräsidien Dokumente zum Ausländerstudium. In Preußen kam bei der polizeilichen Beobachtung von Ausländern dem Berliner Polizeipräsidium eine Schlüsselrolle zu, dessen Beständen im Landesarchiv Berlin Material über politisch verdächtiger Studierende und studentische Organisationen enthalten. Ähnliche Akten der Polizeidirektion München im Staatsarchiv München sind gleichfalls eine hervorragende Quelle zum Ausländerstudium. Im Sächsischen Hauptstaatsarchiv Dresden werden im Bestand „Reichs- und Ausländische Sozialdemokratie“ Berichte sächsischer Polizeidienststellen über politische Aktivitäten unter russländischen und polnischen Studierenden und ihre Kontakte zur deutschen Sozialdemokratie aufbewahrt.

3.3. Im Preußischen Ministerium für Auswärtige Angelegenheiten war die Abteilung Erziehung, Kunst, Wissenschaft und Medizinalwesen in der Hautabteilung III (rechts- und kulturpolitische Fragen) u.a. mit dem zwischenstaatlichen Austausch von Universitäts- und Studienprogrammen sowie der Beschaffung von Material über das ausländische Universitätsund Hochschulwesen befasst und in Diskussionen über die Zulassung von Ausländern zum Studium und zu den Staatsprüfungen (sowie umgekehrt) involviert. Ähnlich ist die Aktenlage in anderen deutschen Staats- und Landesarchiven. Im Archiv des sächsischen Außenministeriums befinden sich mehrere Bestände zur Stellung und zum politischen Verhalten von Ausländern, u.a. auch mit Schriftwechsel zwischen dem Ministerium und der russischen Gesandtschaft. Ein Teil dieser Akten gelangte nach dem Zweiten Weltkrieg - u.a. zusammen mit ähnlichen Beständen aus dem Preußischen Geheimen Staatsarchiv - in sowjetischen Besitz und befindet sich heute im Staatsarchiv für Sozialpolitische Geschichte in Moskau.

3.4. In Preußen und auch in Sachsen schaltete sich im Kontext von Haushaltsberatungen auch das Finanzministerium mehrfach in Diskussionen über die Zulassung von Ausländern 
ein und forderte ihre stärkere Hinzuziehung zur Deckung der Kosten für die Hochschulen. In den entsprechenden Beständen gibt es u.a. Schriftwechsel zu den Hochschuletats, aber auch zu allgemeinen Studentenangelegenheiten und Zulassungsbestimmungen. Für die Zeit nach dem Ersten Weltkrieg kommen in Preußen Etats- und allgemeine Angelegenheiten verschiedener Institute hinzu, die mit dem Auslands- oder Ausländerstudium befasst waren.

4. Das Bundesarchiv und das Archiv des Auswärtigen Amtes. Für die Zeit bis zum Ersten Weltkrieg ist das Material zum Ausländerstudium im Deutschen Reich in den Beständen des ehemaligen Reichsarchivs und des Politischen Archivs des Auswärtigen Amtes aufgrund der geschilderten Kompetenz der deutschen Einzelstaaten nur von ergänzender Bedeutung. Die Akten zur Zulassung ausländischer Bürger zu deutschen Schulen und Hochschulen im Bundesarchiv betreffen vor allem das rechtswissenschaftliche Seminar für russische Staatsstipendiaten an der Universität Berlin. Einzelne Dokumente zur Zulassung zur Prüfung und Anerkennung von Zeugnissen finden sich im Bestand der Rechtsabteilung des Auswärtigen Amtes, zum allgemeinen Pass- und Fremdenpolizeiwesen auch in den Akten des Reichsamtes des Inneren im Bundesarchiv Berlin. Weiteres Material kann in den Beständen zur Deutschen Studentenschaft, zum Verband der Vereine Deutscher Studenten und anderen nichtstaatlichen Institutionen im Bundesarchiv Koblenz recherchiert werden. Im Politischen Archiv des Auswärtigen Amtes werden kultur- und wissenschaftspolitische Fragen für die Zeit vor 1920 in den Beständen der rechts- und handelspolitischen Abteilung sowie der Abteilung Personalien und Verwaltung geführt. Ein Teil der Vorkriegsakten wurde der 1920 geschaffenen Kulturabteilung zugeordnet. Einzelne begleitende Aspekte können u.a. aus Berichten der deutschen diplomatischen Vertretungen im Ausland recherchiert werden. Für konkrete Debatten relevantes Schriftgut befindet sich oft in Form von Abschriften in den Archiven der Länder.

Für Forschungen zur Geschichte des Ausländerstudiums nach 1920 kommt den zentralen staatlichen Archive aufgrund der Veränderungen in der politischen Zuständigkeit eine ungleich größere Bedeutung. Für die Weimarer Republik sind zunächst die Akten der Kulturabteilung des Auswärtigen Amtes für den gesamten Komplex der wissenschaftlichen Beziehungen mit dem Ausland maßgeblich. Hier findet sich u.a. Schriftgut über die Zulassung und Betreuung ausländischer Studenten, die Werbung für ein Studium in Deutschland, das Studium von Deutschen im Ausland, die Arbeit amtlicher und halbamtlicher Stellen bis hin zum Deutschen Akademischen Austauschdienst (DAAD). Eine gute Hilfe für gezielte Recherchen geben die Findbücher zu „Kunst und Wissenschaft" und „Kulturabteilung, revidierte Bestände“. Für die Zeit nach der nationalsozialistischen Machtergreifung wurd die Kompetenzen für das Ausländerstudium neu geordnet und im Wesentlichen im Amt für Wissenschaft des Reichsministerium für Wissenschaft, Erziehung und Volksbildung angesiedelt, wobei das im Bundesarchiv Berlin geführte Schriftgut bereits vor 1933 einsetzt. Von großem Interesse sind hier u.a die Akten des Reichsverbandes der deutschen Hochschulen z.B. mit Schriftgut der Deutschen Akademischen Auskunftsstelle in Dresden und des DAAD. Für das Studium von Russländern in Deutschland, die nach 1922 allerdings mehrheitlich als politische Emigranten zu Staatenlosen wurden und nicht mehr als Bildungsmigranten im eigentlichen Sinne gelten können, sind die Bestände zum Russischen Wissenschaftlichen Institut in Berlin und zu den russischen Emigranten im Politischen Archiv des Auswärtigen Amtes und im Preußischen Geheimen Staatsarchiv relevant. Sie sind u.a. deshalb von Interesse, weil hier auch allgemeine Positionen zum Stellenwert ausländischer Absolventen als Agenten deutscher Interessen diskutiert werden.

5. Personalnachlässe. Hier ist der Kenntnisstand des Autors im Moment auf das Universitätsarchiv Halle sowie das Landesarchiv Berlin, das Preußische Geheime Staatsarchiv und der Handschriftenabteilung der Staatsbibliothek Berlin begrenzt. Von Bedeutung für 
die Untersuchung politischer Grundsatzdebatten um das Ausländerstudium sind hier u.a. die Personalnachlässe von prägenden Persönlichkeiten der deutschen Hochschulpolitik wie F. Althoff und C. H. Becker. In den Universitätsarchiven kann eine Sichtung der Nachlässe von Wissenschaftlern sinnvoll sein, die sich in den Debatten über die ,akademische Ausländerfrage“ stärker engagiert hatten.

6. Gedruckte Quellen. Hier gibt es eine große Vielfalt, aufdie nur schlaglichtartig hingewiesen werden kann. Eine wichtige Quelle sind die stenographischen Protokolle der Landtage. Das Ausländerstudium war hier mehrfach während der Debatten über die Hochschuletats Gegenstand politischer Auseinandersetzungen. Aus den Beiträgen der Abgeordneten und den Stellungnahmen von Vertretern der Kultusministerien lassen sich das Meinungsspektrum in den politischen Parteien und die grundsätzlichen Position der jeweiligen Landesregierungen rekonstruieren. Im Reichstag stand das Thema russländische Studierende z.B. im Jahre 1904 im Mittelpunkt einer politischen Grundsatzdebatte, in der auch der Reichskanzler und der Reichsaußenminister Stellung bezogen. Den Anlass lieferten ein Prozess gegen Schmuggler von revolutionärer Literatur in Königsberg und Kontakte ,anarchistisch“ gesinnter russischer Studenten zur deutschen Sozialdemokratie. Zu diesem Prozess und seinem publizistischen Echo gibt es eine quellenreiche Darstellung von K. Eisner. In bestimmtem Umfang kann auf amtliches oder halbamtliches Schriftgut zurückgegriffen werden. Dazu gehören u.a. Bekanntmachungen im Zentralblatt für die gesamte Unterrichtsverwaltung in Preußen oder im Zentralblatt für das Deutsche Reich, die Jahresverzeichnisse der an den Deutschen Universitäten erschienenen Schriften, die Preußische Statistik mit periodisch veröffentlichten Hochschulstatistiken. In den Münchener Hochschulnachrichten finden sich offizielle amtliche Angaben über Studierendenzahlen, Stellungnahmen von Hochschulen und Ministerien zu Fragen des akademischen Ausländerstudiums, Beiträge des Herausgebers von Salvisberg und von Hochschullehrern. Dem preußischen Kultusministerium nahestehende Auffassungen in Fragen der auswärtigen Kulturpolitik vertrat die Internationale Wochenschrift. Eine vielgestaltige und wertvolle Quelle sind die von amtlichen Stellen, aber auch von studentischen Vereinen oder privat herausgegebene Studien- oder Hochschulführer und Ratgeber für ausländische Studenten. Sie geben nicht nur Auskunft über die Politik gegenüber fremden Studenten, Studienangebote und akademische Vorschriften, sondern spiegeln auch das Selbstverständnis der Autoren bzw. herausgebenden Institutionen wieder. In gedruckter Form verfügbar sind außerdem die Protokolle der Hochschulkonferenzen der deutschen Bundesstaaten und Österreichs 1898 bis 1918. Für statistische und prosopographische Untersuchungen sind, wie bereits erwähnt, weiterhin die halbjährlich gedruckten Adress- und Mitgliederverzeichnisse der Universitäten ergänzend zu den Matrikeln hinzuzuziehen.

7. Zeitgenössische Publizistik. Die zeitgenössische Presse und politische Publizistik spielte in der Debatte über das Auslands- und Ausländerstudium eine zentrale Rolle und ist für historische Forschungen zu diesem Thema in mehrfacher Hinsicht eine hervorragende Quelle. Zunächst informierte sie seit den 1890er Jahren über die Entwicklung der Zahl, der Herkunft und der Verteilung ausländischer Studierender. Neben Parlamentsdebatten, Versammlungen und Vereinen war die Presse das zentrale Medium öffentlicher Meinungsbildung. Auf ihrer Grundlage kann über die Positionen unterschiedlicher gesellschaftlicher Gruppen geurteilt werden. Gleichzeitig war sie ein Mittler zwischen gesellschaftlichen Interessengruppen und staatlicher Politik. Mehrfach fanden deutschlandweite Debatten der „akademischen Ausländerfrage“ statt. In Reaktion auf einen Bericht in der russischen Zeitung „Rossija“ bezogen z.B. im 2. Halbjahr 1912 eine größere Zahl prominenter deutscher Hochschullehrer wie die Mediziner Hertwig und Waldeyer sowie der Historiker Schiemann Stellung zum Ausländerstudium. 
Auf den Einfluss der Presse weisen umfangreichen Sammlungen von Zeitungsausschnitten in Landes- und zum Teil auch Hochschularchiven hin. Da eine Durchsicht aller wichtigen Zeitungen, Wochen- und Monatsschriften sehr aufwendig wäre, empfiehlt sich die Auswertung solcher Pressesammlungen als Ansatz für weitere Recherchen. Einige meinungsführende Zeitungen von gesamtdeutscher und regionaler Bedeutung sind im Anhang genannt. Debatten in Teilöffentlichkeiten wie unter der deutschen Studentenschaft und unter Hochschullehrern sowie Angehörigen der akademischen Berufe sind mangels anderer Quellen vor allem über die Fachoder Verbindungspresse zu recherchieren. Neben der deutschen besaß auch die russländische bzw. russisch-jüdische Studentenschaft eigene studentische Publikationsorgane. Schließlich gibt es so genannte graue Literatur in Form von Broschüren und Streitschriften, die im Umfeld studentischer Vereinigungen sowie berufsständiger und politischer Organisationen entstand. Auch dazu sind im Anhang nähere Angaben zu finden.

B. Forschungsverlauf, Schwerpunkte und Desiderate.* Als selbständiges Problem mit eigenen Fragestellungen und Methoden wird die studentische Migration nach Deutschland erst seit etwas mehr als drei Jahrzehnten behandelt. Bis dahin interessierte das Ausländerstudium eher im Zusammenhang jeweils aktueller politischer Debatten oder im Kontext anderer Fragestellungen. Diese Annäherung - quasi von den Rändern her - bedingte neben der geschilderten Quellensituation einen selektiven Forschungsstand: Während einzelne Aspekte wie das Studium von Russländern, Polen und amerikanischen Studenten bereits häufiger und unter verschiedenen Blickwinkeln thematisiert wurden, bleiben andere weitgehend ein Desiderat. Es fehlen zusammenfassende Würdigungen und die Einordnung in allgemeine Fragen der deutschen und europäischen Hochschulgeschichte sowie des internationalen Kultur- und Wissenstransfers. Auch die Wirkungsgeschichte, der Einfluss auf die Formierung intellektueller Eliten, ist von biographischen Arbeiten und Untersuchungen zu den USA abgesehen noch wenig erforscht. Im Folgenden sollen, von der Genesis des Forschungsproblems ausgehend, zentrale Themen kenntlich gemacht werden.

1. In Deutschland setzte eine über die tagespolitische Polemik hinausgehende systematische Betrachtung des Ausländerstudiums - nach einer ersten Berücksichtigung in Werken von F. Paulsen oder P. Ssymank zur Geschichte des deutschen Hochschulwesens und der deutschen Studentenschaft - in den späten 1920er Jahren ein. Hintergrund größerer Studien von R. Schairer, K. Remme und H. Scurla bildeten konzeptionelle Debatten über eine aktive auswärtige Kulturpolitik des Deutschen Reiches. Diese knüpften an Überlegungen an, die unter F. Althoff um 1900 die Haltung des im preußischen Kultusministerium zum Ausländerstudium beeinflusst hatten, schon am Ende des Ersten Weltkrieges unter veränderten Bedingungen wieder aufgenommen worden waren und in der Weimarer Republik in eine systematische Politik hinüber wuchsen. Auf zentraler Ebene im Reich und in Preußen sind strategische Überlegungen mit Blick auf die kulturelle Außenwirkung Deutschlands wohl als ein zentraler Ansatz für die Politik gegenüber dem Ausländerstudium zu betrachten. Die den Absolventen aus dem Ausland zugedachte Rolle als Agenten des Transfers von ,deutschem Geist“ wurde jedoch von der späteren Forschung noch nicht adäquat thematisiert und spielt auch in den vorliegenden Studien zur auswärtigen Kulturpolitik eine untergeordnete Rolle. So stehen z.B. über die eigentliche „Lamprecht - Bethmann Holweg - Debatte“ hinausgehende Studien zu den Impulsen noch aus, die von den wissenschaftlichen und wissenschaftspolitischen Ideen und Initiativen des Leipziger Universal- und Kulturhistorikers ausgingen und in den 1920er Jahren sowohl publizistische als auch organisatorische Bemühungen zur Förderung des Auslands- und Ausländerstudiums inspirierten. Kaum erforscht - sieht man einmal von der Darstellung Laitenbergers ab - ist die Geschichte des DAAD und seiner Vorläufer. 
2. Ebenfalls schon vor dem Zweiten Weltkrieg wurden in den USA die Einflüsse europäischer Modelle auf die Genesis des amerikanischen Hochschulwesens diskutiert und dabei vor allem unter wirkungsgeschichtlichen Gesichtspunkten auch Fragen des Studiums von Amerikanern in Deutschland untersucht. Erste Publikationen zu diesem Thema erschienen bereits um 1900, wobei besonders D.B. Shumway reichhaltiges prosopographisches Material zusammenstellte, das für die spätere Forschung als Quelle von Bedeutung wurde. In den 1920er und 1930er Jahren entstanden Studien von W. H. Cowley, Ch. F. Thwing und J. A. Walz, die über das bloße Berichten über die Entwicklung des Studiums im Ausland auch seine Wirkung auf das amerikanische Hochschulwesen sowie die Immigration ausländischer Hochschullehrer zum Gegenstand hatten. An solche Forschungen wurde seit Mitte der 1960er Jahren u.a. von C. Diehl, Th. N. Bonner oder Ch. E. McClelland angeknüpft. Methodisch anregend ist K. Jarauschs Aufsatz, der mit prosopographischem Material am Beispiel von Göttingen ein Bild der sozialen und akademischen Dynamik dieser Studentenschaft zeichnet und nach ihrer Spezifik gegenüber den deutschen Kommilitonen fragt. Ein Vergleich zu anderen Studentengruppen, etwa den Russländern, wäre hier lohnend. Auch die Genderforschung (siehe unten) erhielt durch amerikanische Untersuchungen neue Anregungen. P. Mazons Aufsatz z.B. gehört zu den ersten Studien, in denen die Ausländer-, Frauen- und Judenfrage an deutschen Hochschulen im späten Kaiserreich ausdrücklich in ihrer Verknüpfung und im Kontext allgemeiner sozialer Veränderungen in der Studentenschaft und einer Anpassungskrise des deutschen Hochschulsystems betrachtet werden.

3. Auch erste Publikationen über die Verflechtungen zwischen den russischen Studentenkolonien mit dem revolutionären Exil erschienen bereits in den 1920er und 1930er Jahren. Diese enge Betrachtungsweise blieb nicht nur für die marxistische Geschichtsforschung lange dominierend. Den von der zeitgenössischen Publizistik geprägten Blick auf die russischen Studierenden als „Schnorrer, Verschwörer und Bombenwerfer“ quasi aufgreifend, interessierte die sowjetische wie die DDR-Forschung vor allem ihr Anteil am revolutionären Exil und ihre Beteiligung an der antizarischen Bewegung. Da die sowjetische Historiographie außerhalb des revolutionsgeschichtlichen Kontextes Probleme russischen Lebens und russischer Kultur im Ausland überhaupt mied, wurden dem akademischen Studium russischer Untertanen in Deutschland und den damit verbundenen Bildungs- und Kulturtransfers wenig Beachtung geschenkt, so dass die entsprechende Literatur von wenigen Ausnahmen abgesehen (J.N. Ščapov) spärlich blieb. Erst seit den 1990er Jahren wurde der spontane Strom von Studenten an ausländische Hochschulen vom Ende der 1890er Jahre bis zum 1. Weltkrieg in Standardwerken von A. E. Ivanov auch im Kontext der russischen Bildungs- und Sozialgeschichte betrachtet. In der DDR wurde aus hochschul- oder regionalgeschichtlicher Perspektive danach gestrebt, einen Platz in den Annalen der russischen revolutionären Bewegung zu besetzen. Einige so entstandene Studien von B. Brachmann, K. Brundig oder L. Fritsche über russische Studenten in Berlin, Jena und Dresden sind aufgrund der materialreichen Darstellungen oder der Dokumentenanhänge von Interesse. Auch in der Forschung der Bundesrepublik Deutschland wurde das Studium von Russländern im Kontext des Exils untersucht, wobei Arbeiten von D. Dahlmann, L. Schneider oder F. Ortmann den Blick auf breitere historische Zusammenhänge und andere methodische Ansätze weiteten und von der Fokussierung auf die revolutionäre Sozialdemokratie wegführten. C. Weill skizzierte erstmals für den Zeitraum von 1900 bis 1914 ein Gesamtbild der russländischen Studentenschaft im Deutschen Reich und untersuchte sowohl das akademische, soziale, gesellschaftliche wie das politische Leben in den Studentenkolonien. Ihre Studien lieferten maßgebliche Anregungen für das Projekt zur Geschichte des Studiums von Russländern an deutschen Hochschulen an der Universität Halle. Neue Ansätze, die hier verfolgt wurden, sind 
u.a. sozialstatistisch basierte Untersuchungen zur Struktur der russländischen Studentenschaft von A. de Boor, zur Debatte über studenten aus Russland in der deutschen Öffentlichkeit von H.R. Peter, zur nationalen Transformation der russisch-jüdischen Studentenschaft von I. Savinova. Aus der Zusammenarbeit mit französischen, russischen, ungarischen und schweizerischen Wissenschaftlern entstand ein Forschungsnetzwerk, das international vergleichende Studien zur studentischen Migration in und nach Europa anstrebt.

4. Ein weiteres Thema, in dessen Kontext zur Studentenmigration aus Osteuropa intensiver geforscht wurde, ist die jüdische Geschichte und der Antisemitismus. Dies trug zunächst der Tatsache Rechnung, dass Juden unter den ausländischen Studenten im Deutschen Reich bis zum Ersten Weltkrieg eine besonders signifikante Gruppe darstellten. Die „Judenfrage“ an deutschen Hochschulen wurde seit den späten 1970er Jahren in Aufsätzen im Jahrbuch des Leo-BaeckInstituts und verschiedenen Einzelstudien behandelt. Fragen des Ausländerstudiums wurden hier dabei vor allem von J. Wertheimer und später von M. Graetz, P. Mazon, K.H. Pickus, F. Tych, C. Weill aufgegriffen, die z.T. bereits an anderer Stelle angeführt worden sind. Die Ursachen des Phänomens in Russland sind weitgehend erforscht. Spezielle Studien zu den russisch-jüdischen Studenten an deutschen Hochschulen sind jedoch noch rar oder in der Fragestellung begrenzt. Wertheimer konzentriert sich z.B. auf ihre Wahrnehmung im Kontext der „Ausländerfrage“ und des akademischen Antisemitismus und damit ihre besondere Betroffenheit von Diskriminierung, Weill beschreibt die Entstehungsgeschichte russisch-jüdischerer Studentenorganisationen. Erst Graetz, Pickus und zuletzt Savinova richten den Blick auf deren Beitrag zur Konstruktion einer jüdischen Identität und zur Herausbildung einer nationalen Intelligenz, der in Analogie zur Rolle polnischer Studenten im Ausland bei der Formierung der national-polnischen Intelligenz gesehen werden kann.

5. Wie bereits erwähnt, sorgte die Genderforschung in den letzten zwei Jahrzehnten für eine Bereicherung der Literatur zum Ausländerstudium in Deutschland, wobei nicht nur der zeitliche Zusammenfall beider Themen anregend war, sondern auch ihre sachliche Verknüpfung zunehmend thematisiert wird. Das wissenschaftliche Interesse konzentrierte sich vor allem auf russländische und amerikanische Frauen an deutschen und schweizerischen Hochschulen, wobei im Mittelpunkt das Medizinstudium stand. Die Studien von A. Burchardt, S. L. Singer und zuletzt N. Tikhonov sind hier wegweisend, indem sie auf unterschiedliche Weise komparatistisch vorgehen und sowohl die Situation an deutschen und schweizerischen Lehranstalten als auch die Spezifik der amerikanischen, deutschen und russischen Studentinnen in Vergleich setzen. $\mathrm{Zu}$ den Vorzügen der meisten Arbeiten zum Frauenstudium gehören zudem die Dichte des biographischen Materials und die prosopographische Arbeitsweise.

\section{Bibliographischer Anhang}

Die hier angeführte Literatur umfasst den Zeitraum bis 2006, stellt eine Auswahl dar, spiegelt die Forschungsinteressen des Autors wieder und erhebt keinen Anspruch auf Vollständigkeit.

\section{A. GEDRUCKTE QUELLEN}

1. Statistiken, Quelleneditionen: Vom Brocke B., Krüger P. (Ed.) Hochschulpolitik im Föderalismus. Die Protokolle der Hochschulkonferenzen der deutschen Bundesstaaten und Österreichs 1898 - 1918. Berlin 1994; Statistik der Landesuniversitäten: mit Einschluss des Lyceum Hosianum zu Braunsberg, der bischöflichen Klerikalseminare und der Kaiser- 
Wilhelms-Akademie für das Militärärztliche Bildungswesen in Berlin für das Studienjahr 1911/12 (1913), Berlin 1913; Statistik der Landesuniversitäten und Hochschulen: mit Einschluss der Medizinischen Akademie zu Düsseldorf und der Akademie zu Braunsberg, Berlin, 19251926; Eulenburg F. Die Frequenz der deutschen Universitäten von ihrer Gründung bis zur Gegenwart, Leipzig 1904; Titze H. Datenhandbuch zur deutschen Bildungsgeschichte, Bd. 1, T. 1 u. 2. Göttingen 1987,1995

2. Studienführer, Kalendarien, Verzeichnisse: Deutscher Universitätskalender (18721927); Hallescher Universitätskalender; Jahresverzeichnis der an den Deutschen Universitäten erschienen Schriften. Berlin 1885 u. folgende Jahre; Minerva. Jahrbuch der Universitäten der Welt;. Berlin und seine Universität. Ein Führer für Studenten mit besonderer Berücksichtigung der Ausländer, hrsg. von der Amtlichen Akademischen Auskunftsstelle an der Universität Berlin. Berlin 1914; Paszkowskij W. (Ed.) Lesebuch zur Einführung in die Kenntnis Deutschlands und seines geistigen Lebens. Für ausländische Studierende und für die oberste Stufe höherer Lehranstalten des In- und Auslandes, Berlin 1909; Russkij student v Germanii, Avstro-Vengrii i Švejcarii / Russischer Studentenalmanach für Deutschland, Österreich und die Schweiz. III. Jahrgang. Berlin 1912; Margolin D. Studenčeskij spravočnik. Rukovodstvo dlja postupajuščich vo vse vysšie učebnye zavedenija za granicej. Kiev 1909

3. Zeitgenössische Publizistik: Die Ausländerfrage an den Universitäten und Technischen Hochschulen. T. 1-4 / Deutsche Literaturzeitung. No 6-9 (1906), - S.325-331, 389-396, 452-458, 517-521; Bauer E. Russische Studenten. Ein Beitrag zur Geschichte der revolutionären Bewegung in Russland. Annaberg 1901; Von Below G. Die Immatrikulation von Angehörigen feindlicher Staaten / Der Grenzbote. No 73 (1914). - S.328-331; Böninger E. Das Studium von Ausländern auf deutschen Hochschulen. Düsseldorf 1913; Eisner K.(Ed.) Der Geheimbund des Zaren. Der Königsberger Prozess wegen Geheimbündelei, Hochverrat gegen Russland und Zarenbeleidigung vom 12. bis 25. Juli 1904. Nach den Akten und stenographischen Aufzeichnungen mit Einleitungen und Erläuterungen. Berlin 1904; Gerloff H. Die Ausländerfrage an den deutschen Hochschulen. Eine Denkschrift im Auftrage der Vertretung der Deutschen Studentenschaft. Göttingen 1920; Goldschmidt K. Die Ausländer an deutschen Hochschulen und die deutschen Schulen imAusland. / Technik und Wirtschaft, No (1916). - S.245-252; Granovskij L.: O položenii russkoj učaščejsja molodeži, v častnosti - studentov mediciny, v vysšich učebnych zavedenijach Germanii / Obščestennyj vrač. No 8 (1913). S.942-971; Hensing K. Die Ausländerfrage an den deutschen Hochschulen. Vortrag, gehalten auf dem Alldeutschen Verbandstage am 17. Juni 1905 zu Worms. München 1905; Leščinskij J. Iz materialov odnoj studenčeskoj ankety/Russkaja mysl'. No 7 (1914). - S.118-144; Mišilevič D. Položenie russkoj učaščejsja molodežv Germanii. Pis'mo iz Mjunchena. / Obrazovanie. No 9. (Moskau 1901). - S.19-29; Mommsen W. Ausländerstudium / Student und Ausland. No 2-3 (1921) - S.45-47; Sukennikov A. Pervyj kongress russkich studentov i studentok, učaščichsja za granicej. Berlin 1902; Wienecke C. H. Ausländer an deutschen Hochschulen und die „Stimmungen“ ihnen gegenüber / Der Kunstwart und Kulturwart. Halbmonatsschau für Ausdruckskultur auf allen Lebensgebieten. No 26 (1913). - S.168-171

Zeitschriften (Auswahl): Akademische Blätter. Zeitschrift des Verbandes der Vereine Deutscher Hochschulen (Berlin, 1886-1939); Academische Monatshefte (München, 1884-1913); Akademische Rundschau. Zeitschrift für das gesamte Hochschulwesen und die akademischen Berufsstände (Leipzig, 1901-1922); Berliner Akademische Wochenschrift (1906-1907); Burschenschaftliche Blätter (wechselnde Verlagsorte, 1887-1940); Deutsche Literaturzeitung (Berlin, seit 1880); Evrejskij student (Berlin, 1913-1914); Der Freistudent (Halle, 1907-1909); Hochschulnachrichten. Übersicht über das gesamte Hochschulwesen des In- und Auslandes 
(München, 1890-1929); Internationale Wochenschrift für Wissenschaft, Kunst und Technik (Berlin, 1907-1911, dann bis 1921 Internationale Monatsschrift); Der jüdische Student (Berlin, 1904-1933); KC-Blätter. Monatsschrift der im Kartell-Convent der Verbindungen Deutscher Studenten Jüdischen Glaubens vereinigten Korporationen (Berlin, 1910/1911-1933); Kunstwart“ (München, 1887-1932, von 1912-1925 Kunstwart und Kulturwart); Münchner Akademische Rundschau (München, 1911-1915); Otečestvennye zapiski (Berlin, 1912-1914); Studenčeskij listok (Berlin, 1913-1914); Student und Ausland. Monatsschrift zur Förderung der internationalen Zusammenarbeit der Studenten (Leipzig, nachgewiesen 1921); Zeitschrift für angewandte Chemie (Darmstadt, 1887-1931); Zeitschrift für Demographie und Statistik der Juden (Berlin, 1905-1931). Tageszeitungen (Auswahl): Badische Zeitung; Berliner Tageblatt; Deutsche Tageszeitung; Frankfurter Zeitung; Leipziger Neueste Nachrichten; Leipziger Volkszeitung; Münchner Neueste Nachrichten; Nationalzeitung; Neue Preußische (Kreuz-) Zeitung; Norddeutsche Allgemeine Zeitung; Die Post; Saalezeitung; Staatsbürgerzeitung; Der Tag; Tägliche Rundschau; Vorwärts; Vossische Zeitung

\section{B. LITERATUR}

1. Allgemeine und übergreifende Darstellungen. 1.1. Auswärtige Kulturpolitik: Von Brocke B. (Ed.) Das „System Althoff“ in historischer Perspektive. Hildesheim 1991; Vom Bruch R. Weltpolitik als Kulturmission. Auswärtige Kulturpolitik und Bildungsbürgertum in Deutschland am Vorabend des Ersten Weltkrieges. Paderborn 1982; Doeberl M. (Ed.) Das akademische Deutschland. Bd. III: Die deutschen Hochschulen in ihren Beziehungen zur Gegenwartskultur. Berlin, 1930; Düwell K. Deutschlands auswärtige Kulturpolitik 1918 - 1932. Grundlinien und Dokumente. Köln 1976; Düwell, K. Der Einfluss des deutschen technischen Schul- und Hochschulwesens auf das Ausland (1870-1930) / Zeitschrift für Kulturaustausch. No 31 (1981). - S.80-95; Kloosterhuis J. Friedliche Imperialisten. Deutsche Auslandsvereine und auswärtige Kulturpolitik 1906 - 1918. T. 1 u. 2. Frankfurt/Main 1994; Müller G. Carl Heinrich Becker: Internationale Wissenschaft und nationale Bildung. Ausgewählte Schriften. Köln 1997; Müller G. Weltpolitische Bildung und akademische Reform. Carl Heinrich Beckers Wissenschafts- und Hochschulpolitik 1908 - 1930. Köln 1991.

1.2. Ausländerstudium in Deutschland: Drewek P. „Die ungastliche deutsche Universität”. Ausländische Studenten an deutschen Hochschulen 1890-1930 / Jahrbuch für historische Bildungsforschung. No 5 (1999). - S.197-224; Drewek P. Limits of Educational Internationalism: Foreign Students at German Universities Between 1890 and 1930 / Bulletin of the German Historical Institute. No 27 (2000); Laitenberger V. Der DAAD von seinen Anfängen bis 1945 / P. Alter (Ed.) // Der DAAD in der Zeit. Geschichte, Gegenwart und zukünftige Aufgaben. Bonn 2000. - S.21-48; Peter H.R. (Ed.) «Schorrer, Verschwörer, Bombenwerfer?» Studenten aus dem Russischen Reich an deutschen Hochschulen vor dem 1. Weltkrieg. Frankfurt/ Main 2001; Peter H.R., Tikhonov N. (Ed.) Universitäten als Brücken in Europa. Studien zur Geschichte der studentischen Migration [Les universités: des ponts à travers l'Europe. Etudes sur 1'histoire des migrations étudiantes]. Frankfurt/Main 2003; Remme K. Das Studium der Ausländer und die Bewertung der ausländischen Zeugnisse. Berlin 1932; Schairer R. Die Studenten im internationalen Kulturleben. Beiträge zur Frage des Studiums in fremdem Lande. Münster 1927; Schairer R. Ausländische Studenten an deutschen Hochschulen / M. Doeberl (Ed.) // Das akademische Deutschland, Bd. III. - S.523-541; Scurla H. Umfang und Richtung der zwischenstaatlichen Studentenwanderung. Phil D. Leipzig 1933; Plaschka R. G. Mack K. (Ed.) Wegenetze Europäischen Geistes. Wissenschaftszentren und geistige 
Wechselbeziehungen zwischen Mittel- und Südosteuropa vom Ende des 18. Jahrhunderts bis zum Ersten Weltkrieg. München 1983; Plaschka R. G. Mack K. (Ed.) Wegenetze Europäischen Geistes II. Universitäten und Studenten. Die Bedeutung studentischer Migrationen in Mittelund Südosteuropa vom 18. bis zum 20. Jahrhundert. München 1987

2. Hochschulgeschichtliche Arbeiten. 2.1. BERLIN: Brachmann B. Russische Sozialdemokraten in Berlin. Mit Berücksichtigung der Studentenbewegung in Preußen und Sachsen. Berlin 1962; Ergetowski, R. Schiemann-Skandal. Karta z dziejów studenckiej polonii w Berline (1901) / Rozprawy z Dziejów Oświaty. t. XXIV. S.111-141; Feyl O. Die Universität Berlin und das östliche Europa zwischen 1890 und 1933 / G. Oberkofler, E. Zlabinger (Ed.) // Ost-West-Begegnung in Österreich. Festschrift für Eduard Winter zum 80. Geburtstag. Wien/ Köln/Graz 1976. - S.51-71; Hartmann R. Japanische Studenten an der Berliner Universität 1920-1945. Berlin 2003; Heidborn T. Russländische Studierende an der Berliner FriedrichWilhelms-Universität und der Technischen Hochschule Berlin 1880-1914. Ph.D. Bonn 2009

2.2. DARMSTADT: Tych F. Die jüdischen Studenten aus Polen in Darmstadt (1). Biographische Notizen, in: Partner in Dialog / Dokumentation der gemeinsamen Veranstaltung der Technischen Universität Darmstadt mit der Technischen Universität Warschau im Rahmen der VI. Polnischen Musik- und Kulturwoche Darmstadt 1998. Ed. H. Böhme, M. Viefhaus. Darmstadt 1999 (TUD-Schriftenreihe Wissenschaft und Technik. Bd. 78). - S.245-250; Tych F. Die jüdischen Studenten aus Polen in Darmstadt (1). Warum? Welche Folgen? / Dokumentation der gemeinsamen Veranstaltung der Technischen Universität Darmstadt mit der Technischen Universität Warschau im Rahmen der VI. Polnischen Musik- und Kulturwoche Darmstadt 1998. Ed. H. Böhme, M. Viefhaus. Darmstadt 1999 - S.199-208. Viefhaus M. Innensichten: Polnisch-russisch-jüdische Studenten und ihr Umfeld an der TH Darmstadt / Dokumentation der gemeinsamen Veranstaltung der Technischen Universität Darmstadt mit der Technischen Universität Warschau im Rahmen der VI. Polnischen Musik- und Kulturwoche Darmstadt 1998. Ed. H. Böhme, M. Viefhaus. Darmstadt 1999. S.209-244

2.3. GÖTTINGEN: Andreev A.Ju. Die „Göttinger Seele“ der Universität Moskau. Zu den Wissenschaftsbeziehungen zwischen Moskau und Göttingen im frühen 19. Jahrhundert / Jahrbuch für Universitätsgeschichte. No 4 (2001). - S.83-101; Buchloh G., Rix W.T. (Ed.) American Colony of Göttingen. Historical and Other Data Collected Between the Years 18551888. Göttingen 1976; Jarausch K. American students in Germany. 1815-1914: The Structur of German and U.S. Matrikulants at Göttingen University / H. Geitz, J Heideking, J Herbst (Ed.) // German Influence on Education in the United States to 1917. New York 1995. - S.195-211; Maurer T. Balten, Polen, Juden - und strebsame Frauen. Die „russischen“ Studenten Göttingens um die Wende vom 19. zum 20. Jahrhundert, in: Russland und die „Göttingische Seele“ / 300 Jahre St. Petersburg. Ed. by E. Mittler, S. Glitsch. Göttingen 2003. - S.453-473; Lauer R. Die Beziehungen der Göttinger Universität zu Russland / Göttinger Jahrbuch. No 21 (1973). S.219-241; Shumway D.B. The American Students of the University of Göttingen / GermanAmerican Annals. No 8 (1910). - S.171-254

2.3. HALLE: De Boor A. Der Sozialstatus russländischer Studenten an der Universität Halle die Entwicklung einer Kategorie aufgrund von Massendaten. Magisterarbeit. Halle 2003; Kemmelmeier F. Polnische Studentenvereine in Halle 1880-1914) / H.R. Peter N. Tikhonov (Ed.) // Universitäten als Brücken in Europa. - S.279-299; Peter H.R. De Boor A. Klotzsche M. Studenten aus dem Russischen Reich, der „Klinikerstreik“ und die ,akademische Ausländerfrage“ an der Universität Halle vor dem 1. Weltkrieg / H.-J. Rupieper (Ed.) // Beiträge zur Geschichte der Martin-Luther-Universität Halle-Wittenberg 1502-2002. Halle 2002. S.377-406; Peter H.R. Der „Klinikerkampf“ - Studentenprotest und Ausländerproblem an 
der Universität Halle im Winter 1912/1913 / Sachsen-Anhalt. Beiträge zur Landesgeschichte. No 15 (Halle 1999). - S.71-100; Peter H.R. «Hallenser» Sozialrevolutionäre. Eine gruppenbiographische Studie über russländische Studenten in Deutschland 1901-1905 / Jahrbuch für Forschungen zur Geschichte der Arbeiterbewegung. No3 (2004) - - S.17-30; Peter, H.R. Universitet Galle i ego rol' v diskussijach ob obučenii russkich poddanych v Germanii do Pervoj mirovoj vojny / Stranicy istorii. Sbornik nayčnych stat'ej, osvjaščennyj 65-letiju so dnja roždenija professora G.A. Tiškina. St. Petersburg 2008. - S.249-266; Schwabe H. Proteste und Streiks der halleschen Klinikerschaft, in Naturwissenschaften und Medizin im ausgehenden 19. Jahrhundert / Hallesches Symposium 1980 (1). Ed. by W. Kaiser, H. Hübner. Halle 1980. - S.61-67

2.4. HEIDELBERG: Birkenmaier W. Das russische Heidelberg. Zur Geschichte deutsch russischer Beziehungen im 19. Jahrhundert. Heidelberg 1995; Birkenmaier W. Biographisches Lexikon des russischen Heidelberg. 2. Auflage. Heidelberg 1998; Birkenmaier W. Heidelberger Professoren in russischer Sicht. Dokumente aus der Zeit um 1900. Heidelberg 2000; Černjak A. Žurnal russkich studentov v Gejdel'berge / Voprosy literatury. No 1 (1959). - S.172-183; Itenberg B.S. Rossijskaja intelligencija i Zapad. Vek XIX. Očerki. Moskva 1999; Treiber H., Sauerland Heidelberg im Schnittpunkt intellektueller Kreise. Zur Topographie der geistigen Geselligkeit eines Weltdorfes 1850-1950. Oplanden 1995

2.5. JENA: Brundig K. Die Weimar - Jenaer „Iskra“-Tradition. Russische revolutionäre Studenten in Jena 1898-1914. Jena 1977; Feyl O. Beiträge zur Geschichte der slavischen Verbindungen und internationalen Kontakte der Universität Jena. Jena 1960; Hänel Ch. Amerikanische Studierende in Jena zwischen 1850 und 1914 / M. Steinbach, St. Gerber (Ed.) // «Klassische Universität» und «akademische Provinz». Studien zur Universität Jena von der Mitte des 19. bis in die dreißiger Jahre des 20. Jahrhunderts. Jena 2005. - S.498510; Peter H.R. Russländische Studenten in Jena vor dem Ersten Weltkrieg / M. Steinbach, St. Gerber (Ed.) // «Klassische Universität» und «akademische Provinz» Studien zur Universität Jena von der Mitte des 19. bis in die dreißiger Jahre des 20. Jahrhunderts. S. $475-488$

2.6. LEIPZIG: Becker A. How Daring She Was! The ,Female American Colony' at Leipzig University, 1877-1914 / A. Ortlepp, Ch. Ribbat (Ed.) /Taking Up Space. New Approaches to American History. Trier. 2004 - S.31-46; Czok K. Über die politische Betätigung russischer Studenten der Universität Leipzig in den Jahren 1905-1907 / Karl-Marx-Universität Leipzig 1409-1959. Beiträge zur Universitätsgeschichte. Bd. 2. Leipzig 1959. - S.10-22; Ergetowski R. Studenckie organizacje Polaków w uniwersytecie lipskim w latach 1872-1919. Wrocław 1982; Feyl O. Die Aspirantenseminare für Staatsstipendiaten aus Russland an den Universitäten des kaiserlichen Deutschlands / S. Hoyer (Ed.) // Die Geschichte der Universitäten und ihre Erforschung. Theorie - Empirie - Methode. Beiträge der Leipziger Tagung vom 10. und 11. Oktober 1982. Leipzig 1984. - S. 132-143; Gazer H.R. Deutsch-Armenische akademische Beziehungen und der Leipziger „Armenisch-akademische Verein“ / H.R. Peter (Ed.) // «Schorrer, Verschwörer, Bombenwerfer?» Studenten aus dem Russischen Reich an deutschen Hochschulen vor dem 1. Weltkrieg. Frankfurt/ Main 2001. S.169-186; Hoyer S. Studenten aus dem zaristischen Russland an der Universität Leipzig 1870/1914 / H. Lück, B. Schildt (Ed.) // Recht - Idee - Geschichte. Beiträge zur Rechts- und Ideengeschichte für Rolf Lieberwirth anlässlich seines 80. Geburtstages. Köln/Weimar/Wien 2000; Stieda W. Russische Studenten in Leipzig / Neues Archiv für Sächsische Geschichte und Altertumskunde. No 42 (1921). - S.105--123 
2.7. MÜNCHEN: Baur J. Die russische Kolonie in München 1900 1945. Deutsch russische Beziehungenim20.Jahrhundert.Wiesbaden 1998;G.GrimmDieRollederUniversitätMünchen im geistigen Austausch mit den Ländern Südosteuropas zwischen 1826 und 1914 / R.G. Plaschka, K. Mack (Ed.) // Wegenetze Europäischen Geistes. Wissenschaftszentren und geistige Wechselbeziehungen zwischen Mittel- und Südosteuropa vom Ende des 18. Jahrhunderts bis zum Ersten Weltkrieg. München 1983. - S.239-249; Schneider L. Die russische Studentenkolonie und das Echo des revolutionären Russlands in München vor 1914 / Bosl K. u.a. (Ed.) // Bayern im Umbruch. Die Revolution von 1918, ihre Voraussetzungen, ihr Verlauf und ihre Folgen. München/Wien 1969. - S.75-96

2.8. SONSTIGE: Brendel F., Fritsch H. Die Verfolgung fortschrittlicher russischer Studenten in Freiberg (1893-1905). Berlin 1959; Fritsche L. Die Rolle der russischen Studenten an der Technischen Hochschule Dresden in der Zeit von 1900-1914. Phil. D. Dresden 1970; Molik W. Die Polen und die Universität Königsberg in den Jahren 1871 bis 1914 / Nordost-Archiv. N.F. No 3 (1994) - S.427-446; Molik W. Polacy studenci na uniwersytecie w Würzburgu w drugiej połowie XIX i początkach XX wieku / Zapiski Historyczne. No 1(1994). - S.47-71. Siebe D. Ausländische Studenten in Gießen 1900-1949. Akzeptanz, Umwerbung und Ausgrenzung. Gießen 2000

3. Gruppen von Migranten. 3.1. AMERIKANISCHE STUDENTEN: Bernert Ch. Abstract of the Dissertation: Die Wanderjahre: The Higher Education of American Students in German Universities 1870-1914. New York 1984; Bonner Th. N. American Doctors and German Universities. A Chapter in International Intellectual Relations, 1870-1914. Lincoln 1963; Cowley W.H. European Influence on American Higher Education / Education Record No 20. (1939) - S.165-190; Cowley W.H., Williams D. International and Historical Roots of American Higher Education. New York 1991; Diehl C. Americans and German Scholarship. New Haven/Conn. 1978; Faust A. B. The German Element in the United States, with Special Reference to its Political, Moral, Social, and Educational Influence. Vol. 2. Boston/New York 1909. - S.201-249; Fallon D. German Influences on American Education / F. Trommler, E. Shore (Ed.) // The German-American Encounter: Conflict and Cooperation Between two Cultures 1800-2000. New York/Oxford 2001. - S.77-87; Geitz H., Heideking J., Herbst J. German Influence on Education in the United States to 1917. New York 1995. - S.195-211; Herget W. Overcoming the „Mortifying Distance“: American Impressions of German Universities in the Nineteenth and Early Twentieth Centuries / D. Gutzen, W Herget, H.-A. Jacobsen (Ed.) // Transatlantische Partnerschaft. Kulturelle Aspekte der deutsch-amerikanischen Beziehungen. Bonn/Berlin 1992. - S.195-208; Hinsdale B.A. Notes on the History of Foreign Influence upon Education in the United States. Report of the Commissioner of Education for the Year 1897-1898. Washington, D.C. 1899; McClelland Ch.E. German Universities and American Scholars / History of Education Quarterly. No 20 (1980). - S.229-232; Röhrs H. The Classical German Concept of the University and its Influence on Higher Education in the United States. Frankfurt am Main u.a. 1995; Thwing Ch. F. The American and the German University: One Hundred Years of History. New York 1928; Veysey L.R. The Emergence of American University. Chicago 1969; Walz J.A. German Influence in American Education and Culture. Philadelphia 1936

3.2. RUSSLÄNDISCHE STUDENTEN: Andreev A. Ju. Russische Studenten an deutschen Universitäten im 18. und zu Beginn des 19. Jahrhunderts. Überlegungen zu einer sozialen Analyse. / H.R. Peter, N. Tikhonov (Ed.) // Universitäten als Brücken in Europa. Studien zur Geschichte der studentische Migration [Les universités: des ponts à travers l'Europe. Etudes sur l'histoire des migrations étudiantes]. Frankfurt/Main 2003. - S.71-93; 
Bekasova A.B. Studenten aus dem russischen Reich an europäischen Hochschulen im 18. und 19. Jahrhundert: Resultate der Forschung / H.R. Peter (Ed.) // „Schnorrer, Verschwörer, Bombenwerfer?» Studenten aus dem Russischen Reich an deutschen Hochschulen vor dem 1. Weltkrieg. Frankfurt/Main 2001. - S.159-172; Brachmann B. Russische Sozialdemokraten in Berlin. Mit Berücksichtigung der Studentenbewegung in Preußen und Sachsen. Berlin 1962; Dahlmann D. Bildung, Wissenschaft und Revolution. Die russische Intelligencija im Deutschen Reich um die Jahrhundertwende / G. Hübinger, W. Mommsen (Ed.) // Intellektuelle im Deutschen Kaiserreich. Frankfurt/Main 1993. - S.140-157; G. Hausmann Der Numerus clausus für jüdische Studenten im Zarenreich / Jahrbücher für Geschichte Osteuropas. No 41 (1993). - S.509-531; Ivanov A.E. Studenčestvo Rossii konca XIX - načala XX veka. Social'no-istoričeskaja sud'ba, Moskau 1999; Ivanov A.E. Rossijskoe akademičeskoe zarubeže VIII-načala XX veka. / Istočniki po istorii adaptacii rossijskich emigrantov v XIX-XXvv. Sbornik Stat'ej. Mosva 1997; Maurer T. Hochschullehrer im Zarenreich. Ein Beitrag zur russischen Sozial- und Bildungsgeschichte. Köln 1998; Maurer T. Abkommandiert" in die ,akademische Freiheit‘. Russischer Professorennachwuchs in Deutschland im 19. Jahrhundert / D. Diner, F Stern (Ed.) // Deutschland und Russland. Tel Aviver Jahrbuch für deutsche Geschichte. Bd. 24, Gerlingen 1995. - S.63-104;, Peter H. R. „Schnorrer, Verschwörer, Bombenwerfer?“ Zeitgenössische Wahrnehmungsmuster und Stereotype der Betrachtung der Studenten aus Russland in der Forschung / H.R. Peter (Ed.) // „Schorrer, Verschwörer, Bombenwerfer?“. - S.11-32; Peter H.R. Politik und akademisches Ausländerstudium 1905-1913 / H.R. Peter, N. Tikhonov (ed.) // Universitäten als Brücken in Europa. - S.175-194; Peter, H.R. "Tak nazyvaemye Russkie". Izmenenija tipa samosoznanija rossijskich studentov v Germanii posle revoljucii 1905g. / Cahiers du Monde russe. 48 (2007). - S. 427-440; Ščapov Ja. N. Russkie studenty v zapadnoevropejskoj vysšej škole v načale XX v. / Istoričeskie zapiski. No 115 (1987). - S. 193-203 ; Weill C. Convivialité et sociabilité des étudiantes russes en Allemagne 1900-1914 / Cahiers du Monde Russe et Sovietique. No 33 (1991). - S.349-378; Weill C. Etudiantes russes en Allemagne 1900-1914. Quand la Russie frappait aux portes de l'Europe. Paris 1996.

3.3. JÜDISCHE STUDENTEN UND ANTISEMITISMUS: Adler-Rudel S. Ostjuden in Deutschland 1880-1940. Zugleich eine Geschichte der Organisationen, die sie betreuten. Tübingen 1959; Aschheim St. E. Brothers and Strangers. The East European Jews in Germany and German Jewish Consciousness, 1800-1923. Madison/Wis. 1999; Graetz M. Die russisch-jüdischen Studenten an den Universitäten in Deutschland und der Schweiz eine „Subkultur“ um die Jahrhundertwende / M. Graetz, A. Mattioli (Ed.) // Krisenwahrnehmung im fin de siécle. Jüdische und katholische Bildungseliten in Deutschland und der Schweiz. Zürich 1997. - S.139-152; Hoyer S. Die Verbindungen jüdischer Studenten an der Universität Leipzig vor dem 1. Weltkrieg. / GDS-Archiv für Hochschul- und Studentengeschichte. No 5 (2000). - S.51-64; Kampe N. Jews and Antisemites at Universities in Imperial Germany (I). Jewish Students: Social History and Social Conflict" / Year-Book. Leo Baeck Institute, No 30 (1985). - S.357-394; Kampe N. Jews and Antisemites at Universities in Imperial Germany (II). The Friedrich-Wilhelms-University of Berlin: A Case Study on the Students ,Jewish Question / Year-Book. Leo Baeck Institute. No 27 (1982). S.43-101; Peter, H.R., Savinova, I.Russisch-jüdische Studenten in Deutschland - Netzwerke einer sich formierenden intellektuellenElite(1900-1914)/Jahrbuch des Simon-Dubnow-Instituts5(2006). -S.231-253; Pickus K.H. Constructing Modern Identities. Jewish University Students in Germany 18151914. Detroit 1999; Pickus K.H. Jewish University Students in Germany and the Construction of a Postemancipatory Jewish Identity. The Model of the Freie Wissenschaftliche Vereinigung“/ Year-Book. Leo Baeck Institute. No 29 (1984).- S.65-81; Zimmermann M. Jewish Nationalism 
and Zionism in German Jewish Students Organisations / Year-Book. Leo Baeck Institute. No 29 (1984). - S.129-153; Savinova I. Zum Eigenen in der Fremde: Russisch-jüdische Studenten an deutschen Hochschulen von 1881-1914. Magisterarbeit. Halle2005; Wertheimer J. Unwelcome Strangers. East European Jews in Imperial Germany. New York/Oxford 1991; Wertheimer J. The “UnwantedElement”. East European Jews in Imperial Germany/Year-Book. Leo Baeck Institute No 26 (1981). - S.23-46; Wertheimer J. The "Ausländerfrage" at Institutions of Higher Learning. A Controversy over Russian-Jewish Students in Imperial Germany / Year-Book. Leo Baeck Institute. No 27 (1982). - S.187-215; Wertheimer J. Between Tsar and Kaiser. The Radicalisation of Russian-Jewish University Students in Germany / Year-Book. Leo Baeck Institute. No 28 (1983). S.329-349; Weill C. Russisch-jüdische Studentenvereine in Deutschland 1900-1914 / H.R. Peter, N. Tikhonov (Ed.) // Universitäten als Brücken in Europa. - S.229-239

3.4. POLNISCHE STUDENTEN Außer den bereits genannten Arbeiten von Ergetowski, Kemmelmeier, Molik, Tych und Viefhaus vgl. Kochanowskij J.: Die polnische Studentenkolonie in Darmstadt 1894-1914 / Wechselwirkung. Woche der polnischen Kultur und Wissenschaft in der Bundesrepublik Deutschland 25.9. bis 2.10.1990. Darmstadt 1992; Mai J. Der preussische Staat und die polnischen Studenten in Greifswald 1870-1919/Rola Wielkopolski w dziejach narodu polskiego, red. v. St. Kubiak und L. Trzeciakowski. Poznań 1979; Molik W. Die polnischen Studenten und die deutschen Burschenschaften im 19. Jahrhundert / H. Asmus (Ed.) // Studentische Burschenschaften und bürgerliche Umwälzung. Zum 175. Jahrestag des Wartburgfestes. Berlin 1992. - S.254-262.

3.5. ANDERE: Burks A. W. (Ed.) The Modernizers. Overseas Students, Foreign Employees, and Meiji Japan. Boulder/Colorado 1985; Harnisch T. Chinesische Studenten in Deutschland. Geschichte und Wirkung ihrer Studienaufenthalte in den Jahren von 1860 bis 1945 . Hamburg 1999; Hartmann R. Japanische Studenten an der Berliner Universität 1870-1914. Berlin 1997; Nagy J. Ungarische Studenten an der Universität Marburg 1571-1914. Darmstadt 1974; Mouradian, C., Urjewicz, Ch., Weill C. Les étudiantes du Caucase en Allemagne 1900-1914 / Russes, Slaves et Soviétiques, Paris 1992.

4. Frauenstudium: Albicetti J.C. German Influence of the Higher Education of American Women, 1865-1914 / H. Geitz, J. Heideking, J. Herbst (Ed.) // German Influence on Education in the United States to 1917, New York 1995. - S.227-244; Bonner T. N. To the Ends of the Earth. Woman's Search for Education in Medicine, Cambridge/MA 1992; Burchardt A. Blaustrumpf - Modestudentin -Anarchistin? Deutsche und russische Medizinstudentinnen in Berlin 1896-1918. Stuttgart 1997; Burchardt A. „Schwestern reicht die Hand zum Bunde“? Zum Verhältnis zwischen russischen und deutschen Medizinstudentinnen in den Anfängen des Frauenstudiums (1865-1914) / E. Dickmann, E. Schöck-Quinteros (Ed.) // Barrieren und Karrieren. Die Anfänge des Frauenstudiums in Deutschland. Berlin 2000. - S.293-301; Ensele G. Kein Vaterland. Deutsche Studentinnen im Züricher Exil (1870-1908). / A. Schlüter (Ed.) // Pionierinnen. Feministinnen. Karrierefrauen?ZurGeschichte des Frauenstudiums in Deutschland. Pfaffenweiler 1992. - S.9-34. Horn G. (Ed.) Die Töchter der Alma mater Jenensis: neunzig Jahre Frauenstudium an der Universität von Jena. Rudolstadt 1999; Koerner M. Auf fremdem Terrain. Studien und Alltagserfahrungen von Studentinnen 1900 bis 1918. Bonn 1997; Mazon P. Die Auswahl der „,besseren Elemente“: Ausländische und jüdische Studentinnen und die Zulassung von Frauen an deutschen Universitäten 1890-1909. / Jahrbuch für Universitätsgeschichte. No 5 (2002) - S.185-198; Meyer P. They met in Zürich. Nineteenth-Century German and Russian Women Physicians. / L. R. Furst (Ed.) Women Healers and Physicians. Climbing a Long Hill. The University Press of Kentucky 1997; Singer S. L. Adventures Abroad. North American Woman in German-Speaking Universities 1868-1915. Westport/Con./London 2003 
Хартмут Р. П. Джерела та дослідження з історії навчання іноземців у Німеччині. Критичні зауваження щодо ключових питань

https://orcid.org/0000-0001-9331-7290 доктор історичних наук, професор Університет імені Мартіна Лютера міст Галле і Віттенберг Вул. Еміля Абдергальдена 6, 06108, Галле, Німеччина hartmut.peter@geschichte.uni-halle.de

Стаття присвячена аналізу джерел $і$ літератури $з$ історії навчання іноземних студентів у вищих навчальних закладах Німеччини. Метою статті $\epsilon$ виявлення найбільш значущих аспектів цієї проблематики, що своєю чергою дозволить намітити контури подальших досліджень у рамках університетської історії. Відзначається, що тема навчання іноземних студентів в Німеччині, займає важливе місце в політичному дискурсі цієї країни. Незважаючи на це, досліджувана проблематика не знайшла достатнього висвітлення в науковій літературі. Окрема увага приділясться дискусії останньої третини XIX ст. в Німеччині щодо надмірної кількості іноземних студентів в німецьких вищих навчальних закладах. Виникнення цієї дискусії, що отримала назву «akademische Ausländerfrage», було пов'язане з «кризою переповненості» іноземними студентами технічних вищих навчальних закладів і медичних факультетів німецьких університетів. Відзначається, що центральне місце в дискусії займали дві проблеми. Перш за все, звертається увага на націоналістичні та антисемітські групи, які виступали категорично проти великої кількості євреїв зі Східної Свропи серед студентів німецьких вищих навчальних закладів. Крім того, в якості «небажаних іноземців» часто розглядалися польські та російські студенти. Таким чином, дискредитації зазнав принцип інтернаціональності університетів, який був основоположним для академічної спільноти Свропи. Своєю чергою відзначається, що німецькі університети активно протестували проти втручання держави в їх внутрішні справи й автономію загалом. Зокрема німецькі університети виступали категорично проти будь-яких обмежень для іноземних студентів в отриманні ними університетської освіти. Разом із тим університети були зацікавлені в обмеженні кількості студентів, які не мали достатньої підготовки і мотивації. Робиться висновок, що державна політика щодо іноземних студентів однаково протистояла і враховувала позицію німецьких університетів.

Ключові слова: Німеччина, німецькі університети, іноземні студенти, академічне співтовариство.

Хартмут Р. П. Источники и исследования по истории обучения иностранцев в Германии. Критические замечания по ключевым вопросам

https://orcid.org/0000-0001-9331-7290

доктор исторических наук, профессор Университет имени Мартина Лютера городов Галле и Виттенберг Ул. Эмиля Абдергальдена 6, 06108, Галле, Германия hartmut.peter@geschichte.uni-halle.de 
Статья посвящена анализу источников и литературы по истории обучения иностранных студентов в высших учебных заведениях Германии. Целью настоящей статьи является выявление наиболее значимых аспектов этой проблематики, что в свою очередь позволит наметить контуры дальнейших исследований в области университетской истории. Отмечается, что тема обучения иностранных студентов в Германии, занимает важное место в политическом дискурсе этой страны. Несмотря на это, исследуемаяпроблематиканенашладостаточногоосвещения внаучнойлитературе. Отдельное внимание уделяется дискуссии последней трети XIX в. в Германии о чрезмерном количестве иностранных студентов в немецких высших учебных заведениях. Возникновение этой дискуссии, получившей название «akademische Ausländerfrage», было связано с «кризисом переполненности» иностранными студентами технических вузов и медицинских факультетов немецких университетов. Отмечается, что центральное место в дискуссии занимали две проблемы. Прежде всего, обращается внимание на националистические и антисемитские группы, которые выступали категорически против большого количества евреев из Восточной Европы среди студентов немецких высших учебных заведений. Кроме того, в качестве «нежелательных иностранцев» часто рассматривались польские и российские студенты. Таким образом, нападкам и дискредитации был подвергнут сам принцип интернациональности университетов как основополагающий для академического сообщества Европы. В свою очередь отмечается, что немецкие университеты активно протестовали против вмешательства государства в их внутренние дела и автономию в целом. В частности немецкие университеты выступали категорически против любых ограничений для иностранных студентов в получении ними университетского образования. Вместе с тем университеты были заинтересованы в ограничении количества студентов, которые не имели достаточной подготовки и мотивации. Делается вывод, что государственная политика в отношении иностранных студентов в равной степени противостояла и учитывала позицию немецких университетов.

Ключевые слова: Германия, немецкие университеты, иностранные студенты, академическое сообщество. 